\title{
Ensino, Aprendizagem e Uso Profissional da UML em Maringá e Região
}

\author{
Edson OliveiraJr ${ }^{1}$, Thelma E. Colanzi ${ }^{1}$, Aline Amaral ${ }^{1}$, André F. R. Cordeiro ${ }^{1}$, \\ João Choma Neto ${ }^{2}$, Simone R. S. Souza ${ }^{2}$ \\ ${ }^{1}$ Universidade Estadual de Maringá (UEM), Maringá - PR - Brasil \\ ${ }^{2}$ Universidade de São Paulo (USP), São Carlos - SP - Brasil \\ edsonddin.uem.br, teclopesddin.uem.br, ammmamaral@uem.br, \\ cordeiroandrefelipe@gmail.com, joaochoma@usp.br, srociodicmc.usp.br
}

\begin{abstract}
The Unified Modeling Language (UML) has been taught in most of the (under) graduate courses in Computer Science, especially those with an emphasis on Software Engineering. However, little is known about the alignment between how UML has been taught and how it has been used in the software industry. This paper presents the results of two surveys: one with 23 instructors on how UML has been taught in Higher Education Institutions in Maringa and Region and one with 43 practitioners from the same region on how UML has been learned and used in practice. The results are discussed and provide assumptions and directions for improving UML teaching and meeting real market needs.
\end{abstract}

Resumo. A Unified Modeling Language (UML) tem sido ensinada em grande parte dos cursos de (pós-)graduação em Ciência da Computação, especialmente naqueles com ênfase em Engenharia de Software. No entanto, pouco se sabe sobre o alinhamento entre como a UML tem sido ensinada e como ela tem sido utilizada na indústria de software. Este artigo apresenta os resultados de dois surveys: um com 23 docentes sobre como a UML tem sido ensinada em Instituições de Ensino Superior de Maringá e Região e um com 43 profissionais da mesma região sobre como a UML tem sido aprendida e usada na prática. Os resultados são discutidos e fornecem suposições e direções para melhorar o ensino de UML e atender às necessidades reais do mercado.

\section{Introdução}

Maringá, no norte do Paraná, possui mais de 400.000 habitantes e foi eleita recentemente a melhor cidade para morar no Brasil $\left.\right|^{1}$. Além de contar com um arranjo produtivo local compatível com o tamanho da cidade, Maringá possui infraestrutura e qualidade de vida atraente para estudantes e profissionais, principalmente para a área de Tecnologia da Informação (TI). A cidade concentra 12 Instituições de Ensino Superior (IES), contando com mais de 43.000 estudantes em cursos presenciais ${ }^{2}$. Educação e desenvolvimento tecnológico são pilares fundamentais do MasterPlan ${ }^{3}$, um estudo socioeconômico que

1 https://bit.1y/3rcFOwn

2 https://bit.ly/3bT0TFD

$\sqrt[3]{\text { https://bit.ly/3uRpWlg }}$ 
planeja a cidade para até 2047. Maringá é uma cidade que tem investido cada vez mais no desenvolvimento tecnológico da região e do Brasil por meio de um Parque Tecnológico com mais de 400 empresas de TI, o que destaca o papel do desenvolvedor de software, bem como de outros cargos relacionados à indústria de software.

Os processos de desenvolvimento de software são compostos de um conjunto de atividades essenciais, como planejamento, modelagem, desenvolvimento e implementação. Dessa forma, tais processos desempenham um papel central para construção de produtos de software de alta qualidade. Como parte essencial desse processo, a modelagem leva à etapa de implementação do software [Wortmann et al. 2020]. Em 1997 foi proposta uma linguagem para unificar técnicas de modelagem, a Unified Modeling Language (UML). Seu propósito de unificação foi alcançado tornando-se uma linguagem universal até hoje [Petre 2013. Fernández-Sáez et al. 2018].

Pela importância da UML no contexto de desenvolvimento de software existe grande preocupação, por parte dos educadores, de como a UML tem sido absorvida por estudantes e profissionais de software. [Reggio et al. 2014] realizaram um survey com 275 participantes da indústria e academia de vários países sobre quais diagramas UML são usados na prática. Foram considerados diferentes fatores como ambiente de trabalho (academia vs indústria), idade, gênero, nível educacional e experiência. Os diagramas mais utilizados são de classe, de estado, de sequência e de atividade.

[Petre 2013] analisou as razões para a adoção de UML na indústria. O survey coletou informações de 50 empresas. Os resultados indicaram que existem evidências claras de diferentes padrões de uso da UML, o que implica no uso da linguagem em diferentes propósitos e necessidades. Assim, ficou claro que os desenvolvedores estão abertos a conceitos e ferramentas úteis, mas não adotam ferramentas e ideologias em desacordo com suas práticas. Ainda no contexto profissional, [Xavier et al. 2019] conduziram um survey em empresas de software no estado de Minas Gerais para entender o grau de utilização da UML em metodologias ágeis. Os resultados indicaram que cerca de $28 \%$ das empresas usam a UML, sendo os diagramas de caso de uso e de atividade os mais utilizados.

[Choma Neto et al. 2021] avaliaram o nível de adoção da UML em empresas de TI de São Carlos e região. Os resultados indicaram um alto uso da UML, mesmo em empresas que usam metodologias ágeis. Alguns diagramas, como caso de uso, de classe e de atividade são mais adotados. Os resultados oferecem possíveis direcionamentos para melhorar o ensino da UML na região, em especial para apoio ao desenvolvimento ágil.

Os resultados dos trabalhos relacionados motivam fortemente a investigação do ensino, aprendizagem e utilização profissional da UML como linguagem padrão para modelagem de software. Ainda, a modelagem figura como atividade essencial às boas práticas de ES, o que sustenta ainda mais uma investigação do assunto com docentes e profissionais de software. Para tanto, este artigo tem como foco principal apresentar e discutir dois surveys realizados com tal objetivo. Com base nos resultados obtidos, direções sobre o ensino de UML em Maringá e Região são apresentadas e discutidas buscando contribuir efetivamente com o uso profissional de UML. 


\section{Metodologia de Pesquisa}

A metodologia adotada engloba a realização de dois estudos empíricos na forma de surveys ${ }_{4}^{4}$, que seguem as diretrizes de [Linåker et al. 2015]. O primeiro busca responder questões de pesquisa na perspectiva de Docentes: QP1.1: Como a UML tem sido ensinada em Maringá e Região? para entender a modalidade de ensino (presencial, EAD), o tipo de disciplina, carga horária, tipos de diagramas e ferramentas; e QP1.2: Em qual contexto a UML tem sido ensinada em Maringá e Região?, questão relacionada ao tipo de processo de software usado, materiais didáticos, motivação, limitações e percepção de uso na prática.

O segundo survey visa entender como a UML tem sido aprendida e utilizada na prática por Profissionais que trabalham em empresas de desenvolvimento de software. Para isso, as seguintes questões de pesquisa foram elaboradas: QP2.1: Como tem sido a aprendizagem de UML em Maringá e Região? para entender como UML foi aprendida pelos profissionais em relação aos seus diagramas, a forma de aprendizagem (disciplinas específicas ou em outras), carga horária média estudada, ferramentas utilizadas e motivação para a melhoria do ensino; e QP2.2: Como tem sido o uso de UML em empresas de Maringá e Região? para entender como a UML tem sido utilizada na rotina de trabalho dos profissionais, em especial os diagramas utilizados, motivação para usar UML, outras linguagens utilizadas para modelar software e dificuldades para a aplicação de UML na prática. Ao final foram elencadas e discutidas direções e suposições para o ensino e aprendizagem de UML em Maringá e Região.

\section{Ensino de UML na Perspectiva Docente}

O público-alvo do survey foi docentes de IES de Maringá e Região que ministram(ram) disciplinas que incluem UML. A amostragem deste estudo foi realizada por conveniência com contato direto aos docentes de cada IES. Optou-se por um questionário Web 5 por imposições claras da pandemia de coronavírus.

Do total de 23 respondentes, 73,9\% (17) são do sexo masculino e 26,1\% (6) são do sexo feminino. As IES consideradas foram: Fafiman, IFPR Paranavaí, UEM, UFPR Jandaia, UniCesumar, Unifamma, UniFCV, UTFPR Campo Mourão, UNESPAR e FEITEP. Desses, 52,2\% (12) possuem Mestrado, 39,1\% (9) Doutorado e 8,7\% (2) possuem Especialização ou MBA. O tempo como professor de ES foi classificado como: menos de 10 anos (12 - 52,17\%), 10 a 20 anos (7 - 30,43\%) e mais de 20 anos (4 - 17,39\%), tendo como média 11,34 anos e mediana nove anos. Metade da amostra possui mais de nove anos de experiência, o que mostra a sua significância. O tempo de experiência com ensino de UML variou de 1 a 18 anos, com média de 8,26 anos e mediana de nove anos, o que caracteriza uma amostra de docentes experientes no ensino de UML.

\subsection{QP1.1: Forma de Ensino da UML}

Os docentes têm ministrado aulas de UML na sua maioria em cursos presenciais $(82,6 \%$ - 19), seguido por presencial e à distância $(13 \%$ - 3) e somente à distância $(4,3 \%$ - 1). O ensino de UML tem tido forte apreço por cursos presenciais, porém entende-se que a tendência, principalmente após o efeito pandemia de coronavírus, seja um aumento

\footnotetext{
${ }^{4}$ Dados disponíveis em https://doi.org/10.5281/zenodo.4639413

5 https: / / forms.gle/5MVKr5187pwLvUVb9
} 
dos cursos à distância, pela forma de trabalho abstrato realizado no desenvolvimento de software e ferramentas de comunicação remota mais robustas.

Dos 23 docentes, 56,5\% (13) ministram UML como parte de uma disciplina de ES, 26,1\% (6) em uma disciplina específica e 17,4\% (4) diluída em várias disciplinas. Disciplinas específicas para o assunto são raras. Acredita-se que isso se deva ao fato de que a ES evoluiu de forma a englobar muitos temas relacionados ao desenvolvimento de software essenciais para a formação de profissionais que atendam às necessidades do mercado. A carga horária total dedicada à UML variou de 4 a 160 horas/aula, com média de 48,95 horas e mediana de 40. A maioria dos docentes dedicou ao menos 40 horas ao assunto - um valor significativo para uma disciplina semestral de 60 horas. Entende-se, assim, que UML tem sido significantemente ensinada na região de Maringá.

Com relação aos diagramas, os docentes ensinam, principalmente: classe (23), caso de uso (21), sequência (21) e atividade (17). Todos os diagramas da UML foram citados como parte do ensino, porém nem todos são ensinados pelo mesmo docente. De forma absoluta o diagrama de classe já era esperado por causa do seu apelo estático com relação às entidades abstratas de um software e da sua relação direta com a persistência de dados. Assim, é natural pensar neste diagrama em qualquer sistema orientado a objetos.

Desses diagramas, os três com maior ênfase no ensino são: classe (23), caso de uso (19) e sequência (16). Este resultado também já era esperado [Ozkaya and Erata 2020, Fernández-Sáez et al. 2018] pela natureza dos diagramas, especialmente o de caso de uso, pois modela os requisitos funcionais de um sistema e os seus atores. O diagrama de sequência é atrativo, pois representa adequadamente a dinâmica de objetos interagindo, facilitando inclusive a geração de código. Para apoiar o ensino, os docentes usaram as ferramentas: Jude/Astah (21), ArgoUML (5), Draw.io (4), Visio (4) e StarUML (4).

\subsection{QP1.2: Contexto de Ensino de UML}

Com relação ao contexto em que UML é ensinada, 34,8\% (8) docentes indicaram processos tradicionais baseados no modelo cascata ou similar, 13\% (3) ágil (Scrum, etc) e 52,2\% (12) ensinam UML em ambos os contextos. Processos tradicionais têm guiado o ensino de UML, porém a indústria brasileira tem sido pautada por metodologias ágeis [Leal and Santos 2015] e isso é algo que precisa ser repensado urgentemente no ensino de UML. Para tanto, uma análise mais aprofundada do tema pode levar a identificar contribuições pertinentes da linguagem para processos ágeis.

Uma questão importante foi a literatura utilizada pelos docentes. Assim, os seguintes livros foram os mais mencionados: [Larman 2000] (6) e [Guedes 2018] (5). A literatura básica utilizada pelos docentes pode ter efeito direto na forma de ensino de UML e no contexto. O livro de [Larman 2000] é a primeira edição de 2000 e possui exemplos no contexto ágil. O livro de [Guedes 2018] não trata o contexto ágil, mas abrange todos os diagramas da UML com vários exemplos. Entende-se, dessa maneira, que há necessidade de se rever a literatura básica para o ensino de UML, buscando conteúdos recentes e que representem as necessidades da indústria.

Os docentes responderam sobre o que os motiva para ensinar modelagem com UML. Algumas repostas: "proporciona competências e habilidades para a modelagem de sistemas", "amplo entendimento sobre a definição de requisitos", "linguagem padrão facilita a comunicação entre profissionais e expressão de ideias envolvidas no projeto", 
"linguagem aceita na comunidade para descrever arquitetura de software", "referência principal para a modelagem de software", "padronização, melhor comunicação entre os membros da equipe" e "essencial para o desenvolvimento de software". Entende-se, dessa maneira, que tais motivos precisam ser mais explorados considerando a realidade das empresas. Uma possibilidade pode ser o ensino de UML focado em etapas iniciais, como requisitos, e o suporte a arquiteturas de software, incluindo componentes e suas classes com contratos bem estabelecidos das suas interfaces fornecidas e requeridas.

Sobre sintaxe e semântica da linguagem, os docentes afirmam: "linguagem didática adequada para ensinar abstrações", "linguagem padrão para representação da arquitetura de sistemas orientados a objetos", "documentação de código, fluidez. no desenvolvimento e visão de organização futura do código", "linguagem que atende situações simples a complexas, com bom suporte de ferramentas", "capacidade especificar o comportamento não trivial de partes do sistema", "expressividade dos diagramas" e "abordagem gráfica e compreensível do software".

Os docentes também indicaram desafios com o ensino de UML: falta de maturidade na compreensão da abstração necessária no momento do aprendizado, pouco tempo para o ensino detalhado, uso inadequado de alguns diagramas, falta de ferramentas mais intuitivas, falta de interesse dos alunos sem a prática real e a quantidade de diagramas da linguagem a serem ensinados. O problema de falta de maturidade para compreender as abstrações é um desafio constante nos cursos de Computação. Neste sentido, uma estratégia de ensino voltada ao tipo de problema que se quer tratar deve ser traçada, buscando alinhar os diagramas ensinados com cenários reais possíveis, evitando o mal uso ou a subutilização desses diagramas.

Por fim, os docentes foram questionados sobre como percebem o uso de UML na indústria de desenvolvimento de software. Os docentes creem que a UML é importante para a indústria de software atualmente. Apesar de vários trabalhos reportarem o uso massivo da UML na indústria, entende-se que existe uma necessidade dos profissionais entenderem o verdadeiro valor da linguagem por meio de currículos mais voltados para as práticas de mercado. Para docentes e pesquisadores existe uma clara oportunidade de uso da UML, mas que precisa ser repensada para ser melhor compreendida na indústria.

\section{Aprendizagem e Prática de UML na Perspectiva Profissional}

O público-alvo deste survey são egressos ou formandos dos cursos da área de computação que frequentaram as seguintes IES de Maringá e Região: UEM (31), UniCesumar (7), Fafiman (2), UNIPAR (2) e UNESPAR (1). A amostragem deste estudo foi aleatória, obtida por meio da divulgação do questionário Web ${ }^{6}$ nas redes sociais.

Do total de 43 respondentes, 46,5\% (20) possuem Graduação, 23,3\% (10) Mestrado, 20,9\% (9) possuem Especialização ou MBA, e 9,3\% (4) são graduandos. O tempo de experiência, graduação mais profissional, variou entre 2 e 25 anos, com média de 10,6 anos e mediana 10. Mais da metade dos profissionais possui ao menos dez anos de experiência, caracterizando uma amostra significativa de profissionais experientes, que exercem diferentes cargos nas empresas em que atuam. Os cinco cargos mais mencionados e seus percentuais são: Desenvolvedor 32,6\% (14), Analista de Sistemas 20,9\% (9), Analista de Teste 4,7\% (2), Programador Pleno 4,7\% (2) e CEO 4,7\% (2).

\footnotetext{
6htps://forms.gle/YoReTkW3JiDaVcgy5
} 


\subsection{QP2.1: Aprendizagem de UML}

Dos 43 profissionais, 60,5\% (26) aprenderam UML como parte de uma disciplina de ES, 27,9\% (12) aprenderam UML de forma diluída entre várias disciplinas, e 11,6\% (5) em uma disciplina específica ao assunto. A média de carga horária total dedicada ao ensino de UML na formação dos profissionais foi de 102,8 horas, com de mediana 60 horas.

Com relação aos diagramas UML, os profissionais indicaram os três que tiveram maior contato na graduação: classe (39), caso de uso (39), sequência (24), componentes (8), estados (7), atividades (4), objetos (2) e visão geral de integração (1). É interessante observar que os diagramas mais vistos são também os mais frequentemente citados pelos docentes. Dentre as ferramentas CASE utilizadas como apoio, as mais citadas foram: Jude/Astah (32), Draw.io (12), Lucid (7), StarUML (2). Cinco profissionais responderam que não aprenderam ferramentas de modelagem UML durante a graduação.

Os profissionais responderam a uma pergunta aberta a respeito do que deveria mudar/melhorar no ensino de modelagem UML. Os principais pontos citados foram: uso de exemplos reais (19), aumentar as atividades práticas (10), foco nas necessidades do mercado (8). Dentre os profissionais que apontam a necessidade de utilizar exemplos reais da indústria em sala de aula, o profissional $\mathrm{P} 21$ sugere "trazer casos reais durante o ensino... o ensino visto em sala e a prática no dia-a-dia diferem consideravelmente. Principal aspecto é quando utilizar cada recurso e o porquê". Além disso, P11 argumenta: "Apenas praticamos com casos fictícios criados para fins didáticos, o que não transmite ao aluno uma sensação de confiança e aprendizado de fato".

Além dos projetos reais, mais de $25 \%$ dos respondentes sugeriram aumentar as atividades práticas de modelagem UML nas disciplinas, como por exemplo, P22: "Aumentar as atividades onde os alunos têm que criar o próprio diagrama.". O profissional P41 sugere "codificar o projeto criado em UML para que o aluno veja mais de perto a relação entre a modelagem e a implementação final.". Além disso, é necessário utilizar alguma ferramenta CASE para as atividades práticas como sugerido por P43 que se formou em 2010 e não estudou nenhuma ferramenta: "Poderia focar em alguma ferramenta CASE para sua utilização".

Vários profissionais que sugeriram concentrar o ensino nas necessidades do mercado, alegam que seria mais interessante aprofundar o ensino nos diagramas UML utilizados na indústria de software, como evidenciado por P33 "O ensino de modelagem UML deveria focar mais no que é realmente relevante e utilizado no mercado e no dia-a-dia do desenvolvimento e planejamento de software, ao invés de tentar cobrir e ensinar uma série de diagramas e modelagens que não tem aplicação real." Outras sugestões menos frequentes incluem atrelar o ensino de UML à aplicação dos princípios SOLID de arquitetura de software, ensinar UML de maneira transversal com as demais disciplinas da computação, evidenciar as vantagens de aprender UML para motivar os alunos, e a atualização dos docentes em relação às demandas da indústria de software.

Deste modo, os dados apresentados caracterizam como a aprendizagem de UML se dá em Maringá e Região. Considerando que ao menos $64 \%$ dos profissionais que responderam a este survey se formaram na última década e as sugestões dos respondentes para melhorar o ensino de UML, pode-se inferir que o ensino de UML se dá muitas vezes utilizando exemplos fictícios e com menos atividades práticas do que o desejado 
pelos profissionais enquanto alunos. A questão da quantidade de atividades práticas está relacionada ao fato da maioria dos respondentes ter estudado UML como parte de uma disciplina de Engenharia de Software, o que restringe a quantidade de atividades práticas que o docente pode incluir na disciplina.

Em consonância com as respostas dos docentes, a ferramenta Jude/Astah foi a mais indicada pelos profissionais e os três diagramas mais estudados pelos profissionais foram o de classe, o de caso de uso e o de sequência. Em geral, 7 diagramas UML foram estudados por ao menos 25 profissionais. Por outro lado, 8 profissionais afirmaram que o ensino de UML deveria ser mais focado nas necessidades do mercado, logo eles acreditam que aprenderam mais diagramas UML do que o necessário. Isso mostra que os docentes deveriam enfatizar mais durante o ensino a importância UML para a indústria de software.

\subsection{QP2.2: Uso de UML nas Empresas}

Dos 43 participantes, 40 foram considerados para a análise e discussão sobre como a UML tem sido praticada nas empresas, uma vez que o escopo de aplicação do survey foi em empresas de Maringá e Região, e três participantes não atenderam a este pré-requisito.

Considerando o método de desenvolvimento de software adotado pelas empresas de Maringá e Região em que os profissionais atuam, 68\% (27) utilizam métodos ágeis, $2 \%$ (1) utilizam os métodos tradicionais e 30\% (12) utilizam ambos. Os métodos mais citados foram: Scrum (11), Kanban (7), Scrum com Kanban (5), Ágil (3), Nenhum (3), Tradicional (2) e Lean (2).

Com relação à experiência com o uso profissional da UML, a distribuição percentual dos profissionais foi: 35\% (14) têm experiência básica com UML, 30\% (12) moderada, 5\% (2) avançada. Os demais 30\% (12) afirmam nunca terem modelado software com UML. Levando-se em conta os diagramas UML, os profissionais utilizam mais: classes (27), casos de uso (23), componentes (12), sequência (11), atividades (8), estado (7), comunicação (6), visão geral de interação (4), implementação (3), tempo (2), estrutura composta (2) e objetos (2). Observa-se que os 5 diagramas mais frequentes são também os mais citados pelos docentes e os mais aprendidos pelos profissionais.

Os profissionais responderam a uma pergunta aberta com relação à motivação da empresa em usar UML. Os principais pontos citados foram: entendimento do projeto (23), utilização de linguagem padrão (7), importância da documentação (6), qualidade (2), auditoria do sistema (1) e requisição do cliente (1).

Vários profissionais alegam que a UML auxilia no entendimento do projeto, incluindo seu planejamento, definição do fluxo de tarefas, do escopo e dos requisitos. Tais pontos podem ser observados em repostas como as dos profissionais P17 -"Exemplificação de estrutura técnica em alto nível para demonstrar dependências e pontos de melhora. Também para demonstrar para pessoas não técnicas alguma dificuldade técnica enfrentada pela equipe" e P29 -"Melhora a interpretação dos requisitos e comunicação entre os analistas e desenvolvedores". Um outro ponto também ressaltado pelos profissionais refere-se ao fato da UML ser uma linguagem de especificação padrão e consequentemente estabelecer um canal padrão de comunicação entre os desenvolvedores, isto pode ser observado em respostas como as dos profissionais P21 -"Estabelece uma linguagem única entre desenvolvedores e analistas..." e P39 - "A facilidade como uma informação pode ser transmitida/absorvida pelos membros equipe". 
Uma fonte importante de motivação é o uso da UML para documentação dos artefatos produzidos ao longo do desenvolvimento de software. Respostas como as dos profissionais P31 -"Documentação visual gera muito mais valor e consegue repassar o conhecimento muito mais fácil do que várias páginas de documentação.” e P35 "Documentação mais completa" nos permitem observar tal fonte.

Em uma última questão aberta, os profissionais relataram dificuldades quanto ao uso da UML. Os principais pontos citados foram: falta de conhecimento dos profissionais (13), falta de tempo e valorização (10), UML não é necessária (3), dificuldade de manter a documentação atualizada (3), não é interessante para pequenas empresas (2), falta de boas ferramentas de modelagem (2), não aprendeu direito na faculdade (2), falta de cargos específicos (1). Os dois pontos mais citados serão detalhados a seguir.

Quanto à falta de conhecimento em UML ressaltada pelos profissionais, respostas como as dos profissionais P2 -"No meu caso, a falta dela é a grande dificuldade" e P7 -"A minha é a falta de conhecimento para poder argumentar com gestores e mostrar o real valor entregado" demonstram claramente este problema. Os profissionais também destacaram a falta de tempo e valorização do trabalho de documentação pelas empresas. Isso é retratado em respostas como: "...Dificilmente existe um reconhecimento para atividades de documentação. Sem uma motivação real e o pagamento efetivo por esse trabalho, o profissional tende a se dedicar em realizar o trabalho que agrega valor ou que pode ser mensurado" (P3) e "Muito tempo dispendido para pouca vantagem" (P18).

Deste modo, os dados apresentados caracterizam como o uso da UML se dá em empresas de Maringá e Região. Pode-se observar que a maioria dos entrevistados tem experiência básica (35\%) ou moderada (30\%) com relação à UML, o que é retratado pelos diagramas mais utilizados (classes e casos de uso) e demonstra uma consonância entre os diagramas que recebem maior atenção no processo de ensino-aprendizagem. Além disso, a grande maioria das empresas não adota outras linguagens de especificação, apesar de algumas menções a representações como fluxograma e diagrama de fluxo de dados.

Com relação às principais motivações para o uso da UML, observa-se que estas são associadas a questões de natureza técnica, uma vez que a UML permite um melhor entendimento do projeto, além de estabelecer um padrão de modelagem e de comunicação entre os desenvolvedores. Os profissionais apresentam como principal limitação para o uso de UML, a falta de conhecimento dos profissionais com relação à UML. Esta questão pode estar associada com a demanda excessiva por profissionais de TI, o que faz com que as empresas contratem profissionais que ainda não tenham sua formação completa e, por isso, pouco ou nenhum conhecimento em modelagem de software.

\section{Suposições e Direções para o Ensino de UML}

A partir dos resultados obtidos com os surveys foram delineadas suposições e direções, apresentadas a seguir, para o ensino de UML buscando melhorar a qualidade do ensino, a motivação dos alunos e o alinhamento entre a academia e a indústria de software.

Suposição \#1: docentes com nível de Mestrado tendem a ser a maioria no ensino de UML em Maringá e Região. Como Direção motiva-se a formação de mestres em Ciência da Computação e sua capacitação com estágios de docência e em empresas de software.

Suposição \#2: cursos EAD tendem a crescer nos próximos anos, principalmente por 
causa das experiências positivas com a situação imposta pela pandemia de coronavirus, especialmente para a área de TI em Maringá e Região. Como Direção para isso, sugere-se a alta capacitação dos docentes nesta modalidade de ensino em um curto prazo de tempo.

Suposição \#3: UML tem sido ensinada majoritariamente em disciplinas de ES, o que dificulta a sua relação com disciplinas de práticas de programação. Como Direção a UML pode ser ensinada como parte do programa de disciplinas como, por exemplo, Programação Orientada a Objetos e Paradigmas, Linguagens de Programação, Padrões de Projeto e de Arquiteturas, buscando alinhar o poder de abstração dos diagramas com os recursos das linguagens de implementação de software.

Suposição \#4: o ensino de UML aborda diversos diagramas que são pouco utilizados na prática. Como Direção espera-se que o ensino de UML se concentre nos diagramas de caso de uso, classe e sequência dedicando-se mais horas para esses com exemplos reais e práticos de situações rotineiras nas empresas.

Suposição \#5: a UML é contextualizada majoritariamente em processos tradicionais e fictícios. Como Direção são necessários exemplos de casos reais de ambientes ágeis que tangem as necessidades reais do mercado. Para isso, parcerias entre as IES e as empresas podem facilitar e contribuir efetivamente com a qualidade do ensino. Uma alternativa pode ser o uso de projetos de software livre como uma forma de propiciar exemplos reais e de atuação em projetos reais aliados ao ensino de UML.

Suposição \#6: a literatura adotada para o ensino de UML é satisfatória, mas carece de imersão em ambientes ágeis. Como Direção sugere-se a adoção de artigos de conferências e periódicos que relatam exemplos de aplicação da UML em contextos ágeis.

Suposição \#7: docentes são muito bem motivados para o ensino de UML, mas não compreendem efetivamente as necessidades do mercado. Como Direção sugere-se a realização de estudos empíricos em empresas de software (por ex., surveys) para capturar a essência das necessidades reais do mercado e atrelá-las aos recursos da UML.

Suposição \#8: os docentes que ensinam UML enfrentam dificuldades em transmitir aos discentes conceitos relacionados à abstração, necessários para propor uma solução computacional em nível de modelagem UML a partir de um problema do mundo real. Como Direção sugere-se a possibilidade de cooperação com outros docentes de disciplinas dedicadas às práticas de programação, visando materiais didáticos interligados, com exemplos comuns e reais para tais disciplinas.

Suposição \#9: a maioria dos profissionais tem conhecimento básico ou moderado de UML. Como Direção sugere-se que as empresas forneçam aos seus colaboradores cursos de capacitação em linguagens de modelagem, como a UML. Tais cursos podem ser propostos pelas IES na forma de extensão universitária envolvendo alunos de (pós-)graduação.

Suposição \#10: a falta de tempo e de valorização da documentação é um fator limitante para o uso da UML pelas empresas. Como Direção sugere-se o suporte aos profissionais das práticas de gerência de projetos de software para a definição de cronogramas mais efetivos, o que pode fazer com que as empresas dediquem mais tempo e se beneficiem com as atividades de modelagem com o apoio da UML. 


\section{Considerações Finais}

Como contribuições principais deste artigo tem-se: (i) dados atuais sobre ensino, aprendizagem e uso profissional de UML em Maringá e Região; e (ii) pontos de reflexão como direções sobre o ensino de UML guiados por formas e contextos em que UML é ensinada por docentes, como o seu aprendizado é percebido e como o seu uso acontece atualmente na perspectiva de profissionais de empresas de software.

Como possibilidade de trabalhos futuros vislumbram-se: (i) a expansão do segundo survey para profissionais que atuam em empresas de software, independentemente de estarem situados em Maringá e Região; (ii) a produção de material didático considerando as direções apresentadas neste artigo para a academia e a indústria de software com licença aberta nos moldes Creative Commons; e (iii) criação de um curso de extensão que possa abranger docentes, discentes e profissionais de Maringá e Região buscando suprir necessidades pontuais do ensino de UML.

\section{Agradecimentos}

Os autores agradecem enormemente aos docentes e profissionais pelo precioso tempo e ao Prof. R. Patroni da Software by Maringá pelo apoio na divulgação desta pesquisa.

\section{Referências}

Choma Neto, J., Bento, L. H. T. C., OliveiraJr, E., and Souza, S. R. S. (2021). Are we teaching UML according to what IT companies need? A survey on the São Carlos - SP region. In EduComp, pages 34-43. SBC.

Fernández-Sáez, A. M., Chaudron, M. R., and Genero, M. (2018). An industrial case study on the use of UML in software maintenance and its perceived benefits and hurdles. Empirical Softw. Engg., 23(6):3281-3345.

Guedes, G. (2018). UML 2 - Uma Abordagem Prática. Novatec Editora.

Larman, C. (2000). Utilizando UML e Padrões. Grupo A - Bookman.

Leal, T. and Santos, G. (2015). Um survey sobre métodos Ágeis e o pós-agilismo. In CibSE, pages 53-66.

Linåker, J. et al. (2015). Guidelines for conducting surveys in software engineering. Technical Report 1.1, Lund University, Sweden.

Ozkaya, M. and Erata, F. (2020). A survey on the practical use of UML for different software architecture viewpoints. Information and Software Technology, 121:106275.

Petre, M. (2013). UML in practice. In ICSE, pages 722-731. IEEE.

Reggio, G., Leotta, M., and Ricca, F. (2014). Who knows/uses what of the UML: A personal opinion survey. In MODELS, pages 149-165. Springer.

Wortmann, A., Barais, O., Combemale, B., and Wimmer, M. (2020). Modeling languages in Industry 4.0: an extended systematic mapping study. Software and Systems Modeling, 19(1):67-94.

Xavier, A., Martins, F., Pimentel, R., and Carvalho, D. (2019). Aplicação da UML no contexto das metodologias ágeis. In ENCOMPIF, pages 1-8. SBC. 